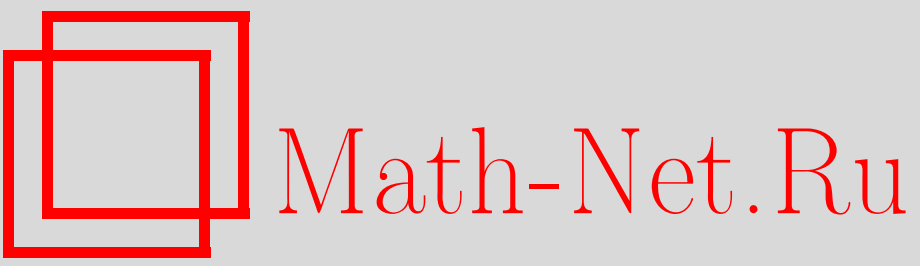

Ф. М. Лев, Безмассовые элементарные частицы в квантовой теории над полем Галуа, ТМФ, 2004, том 138, номер 2, 246-268

DOI: https://doi.org/10.4213/tmf24

Использование Общероссийского математического портала Math-Net.Ru подразумевает, что вы прочитали и согласны с пользовательским соглашением

http://www . mathnet.ru/rus/agreement

Параметры загрузки:

IP: 3.85 .183 .62

26 апреля 2023 г., 14:51:17 
ТЕОРЕТИЧЕСКАЯ

И МАТЕМАТИЧЕСКАЯ

ФИЗИКА

Том 138, № 2

февраль, 2004

(C) 2004 г.

Ф. М. Лев*

\section{БЕЗМАССОВЫЕ ЭЛЕМЕНТАРНЫЕ ЧАСТИЦЫ В КВАНТОВОЙ ТЕОРИИ НАД ПОЛЕМ ГАЛУА}

Рассмотрены безмассовые элементарные частицы в квантовой теории над полем Галуа (КТПГ). Ранее нами было показано, что теория обладает новой симметрией между частицами и античастицами, не имеющей аналога в стандартном подходе. В данной статье доказано, что эта симметрия согласуется со всеми операторами, описывающими безмассовые частицы. Как следствие спин безмассовых элементарных частиц может быть только полуцелым (в обычных единицах), а сушествование безмассовых нейтральных элементарных частиц несовместимо с теоремой о связи спина со статистикой. Это, в частности, означает, что в КТПГ фотон и гравитон могут быть только составными частицами.

Ключевые слова: поля Галуа, безмассовые частицы, модулярные представления.

\section{1. ВВЕДЕНИЕ}

В различных реакциях при высоких энергиях возможны рождение и аннигиляция произвольной частицы другими частицами и, следовательно, понятия элементарной и составной частиц не имеют ясного экспериментального смысла. В то же время теоретически эти понятия хорошо определены. Частица называется элементарной, если полный набор ее волновых функций образует пространство неприводимого представления (НП) группы или алгебры симметрии данной теории. Такой подход был впервые предложен Вигнером в работе [1], где были построены унитарные НП группы Пуанкаре.

В рамках стандартного подхода к построению квантовой теории каждая элементарная частица либо имеет, либо не имеет соответствующей античастищы с той же массой и спином. В последнем случае можно считать, что частица совпадает со своей античастищей. Элементарные частицы с таким свойством называются нейтральными.

Обсудим вкратце, как стандартная теория объясняет существование частиц и античастиц. Рассмотрим, например, электрон и позитрон, которые являются античастицами друг для друга. Объяснение основано на том, что уравнение Дирака имеет решения как с положительными, так и с отрицательными энергиями. Как заметил Дирак (см.,

*E-mail: felixlev@hotmail.com 
например, его нобелевскую лекцию [2]), существование решений с отрицательной энергией представляет собой трудность, требуюшую разрешения. В стандартном подходе решение достигается с помошью процедуры вторичного квантования, в которой операторы рождения и уничтожения позитрона имеют обычный смысл, но входят в квантовый лагранжиан с коэффициентами, представляюшими решения с отрицательной энергией. Таким образом, реализуется идея о том, что рождение или уничтожение античастишы можно рассматривать соответственно как уничтожение или рождение соответствующей частищы с отрицательной энергией. Тем не менее эта идея реализуется скорее неявно, чем явно, поскольку отрицательные энергии не имеют непосредственного физического смысла в стандартной теории. Отметим также, что электрон и позитрон описываются унитарными НП с положительной энергией, но эти представления полностью независимы. В то же время НП с отрицательной энергией вообще не используются.

В работах [3] нами был предложен подход к построению квантовой теории, в котором волновые функции рассматриваемой системы описываются элементами линейного пространства над полем Галуа, а операторы физических величин - линейными операторами в этом пространстве. Подробное обсуждение этого подхода содержится в недавней работе [4]. В ней, в частности, показано, что при некоторых условиях такое описание приводит к тем же предсказаниям, что и стандартный подход, а также приведены аргументы в пользу того, что описание квантовых систем в терминах полей Галуа является более естественным, чем стандартное описание в терминах комплексных чисел.

Первый очевидный вывод, который можно сделать о квантовых теориях над полем Галуа (КТПГ), заключается в том, что поскольку любое поле Галуа имеет лишь конечное число элементов, то в КТПГ в принципе не может быть расходимостей, а все операторы автоматически корректно определены. Также естественно ожидать, что КТПГ обладает рядом свойств, не имеющих аналога в стандартной теории, поскольку арифметика полей Галуа отличается от стандартной арифметики.

В частности, как показано в [4], в КТПГ частица и ее античастица описываются одним и тем же НП алгебры симметрии, в отличие от стандартного подхода, в котором частица и ее античастица описываются независимыми НП группы симметрии. Этим автоматически объясняется существование античастиц и показывается, что частица и ее античастица представляют собой различные состояния одного и того же объекта. Следовательно, КТПГ обладает новой симметрией между частицами и античастицами, не имеющей аналога в стандартной квантовой теории. Вместе с тем возникает вопрос о том, являются ли нейтральные частищы элементарными или лишь составными.

Проблема сушествования нейтральных элементарных частиц представляет наибольший интерес в случае безмассовых частиц, например в случае фотона или гравитона. Поэтому в данной статье (см. разделы 2 и 3 ) рассматривается безмассовый случай. Новая симметрия подробно описана в разделе 4 , а в разделе 5 показано, что условие сушествования вакуума является непротиворечивым лишь для частиц с полуцелым спином (в обычных единицах). В разделе 6 мы доказываем, что указанная симметрия согласо- 
вана с операторами представления для безмассовых частиц. В разделе 7 показано, что как следствие в безмассовом случае сушествование нейтральных элементарных частиц в КТПГ несовместимо со стандартной связью спина со статистикой.

Хотя понятие поля Галуа является крайне простым и элегантным, большинство физиков не знакомы с этим понятием. Поэтому в работе [4] была предпринята попытка объяснения основных фактов, касающихся полей Галуа, при помоши аргументов, которые, как мы надеемся, могут быть восприняты физиками. Читатели, не знакомые с полями Галуа, также могут почерпнуть основные сведения из стандартных учебников (см., например, [5]).

\section{2. ОПЕРАТОРЫ ПРЕДСТАВЛЕНИЯ АЛГЕБРЫ АНТИ-ДЕ СИТТЕРА}

Если обычная квантовая теория обладает группой (или алгеброй) симметрии, то сушествует унитарное представление этой группы (или представление алгебры эрмитовыми операторами) в гильбертовом пространстве, описывающем рассматриваемую квантовую систему. В данной статье предполагается, что алгебра симметрии является аналогом алгебры анти-де Ситтера (AdS) so(2,3), а квантовые системы описываются представлениями этой алгебры в пространствах над полем Галуа (подробности см. в [4]). Стандартная группа AdS, так же как и группа Пуанкаре, является десятипараметрической, но, в отличие от группы Пуанкаре, все ее генераторы представления отвечают угловому моменту. В работе [4] мы объяснили, почему для наших целей удобно работать в системе единиц $\hbar / 2=c=1$. В этом случае генераторы представления безразмерны и их коммутационные соотношения могут быть записаны в виде

$$
\left[M^{a b}, M^{c d}\right]=-2 i\left(g^{a c} M^{b d}+g^{b d} M^{c d}-g^{a d} M^{b c}-g^{b c} M^{a d}\right),
$$

где $a, b, c, d$ принимают значения $0,1,2,3,5$, а операторы $M^{a b}$ антисимметричны. Компоненты диагонального метрического тензора равны $g^{00}=g^{55}=-g^{11}=-g^{22}=$ $-g^{33}=1$. В этих единицах фермионы имеют нечетный, а бозоны - четный спин. Если спин частицы равен $s$, то соответствующее НП алгебры $s u(2)$ имеет размерность $s+1$. Заметим, что при такой интерпретации $s$ не зависит от выбора системы единиц (в отличие от максимального собственного значения $z$-проекции оператора спина).

При анализе НП, реализуюших соотношения (1), удобно работать с другим набором из десяти операторов. Пусть $\left(a_{j}^{\prime}, a_{j}^{\prime \prime}, h_{j}\right), j=1,2,-$ два независимых набора операторов, удовлетворяющих коммутационным соотношениям

$$
\left[h_{j}, a_{j}^{\prime}\right]=-2 a_{j}^{\prime}, \quad\left[h_{j}, a_{j}^{\prime \prime}\right]=2 a_{j}^{\prime \prime}, \quad\left[a_{j}^{\prime}, a_{j}^{\prime \prime}\right]=h_{j}
$$

Эти наборы независимы в том смысле, что при разных $j$ они взаимно коммутируют друг с другом. Оставшиеся четыре оператора обозначим через $b^{\prime}, b^{\prime \prime}, L_{+}, L_{-}$. Операторы $L_{+}, L_{-}$имеют следуюший смысл. Операторы $L_{3}=h_{1}-h_{2}, L_{+}, L_{-}$удовлетворяют 
коммутационным соотношениям алгебры $s u(2)$

$$
\left[L_{3}, L_{+}\right]=2 L_{+}, \quad\left[L_{3}, L_{-}\right]=-2 L_{-}, \quad\left[L_{+}, L_{-}\right]=L_{3}
$$

а остальные коммутационные соотношения имеют вид

$$
\begin{aligned}
{\left[a_{1}^{\prime}, b^{\prime}\right] } & =\left[a_{2}^{\prime}, b^{\prime}\right]=\left[a_{1}^{\prime \prime}, b^{\prime \prime}\right]=\left[a_{2}^{\prime \prime}, b^{\prime \prime}\right]= \\
& =\left[a_{1}^{\prime}, L_{-}\right]=\left[a_{1}^{\prime \prime}, L_{+}\right]=\left[a_{2}^{\prime}, L_{+}\right]=\left[a_{2}^{\prime \prime}, L_{-}\right]=0, \\
{\left[h_{j}, b^{\prime}\right] } & =-b^{\prime}, \quad\left[h_{j}, b^{\prime \prime}\right]=b^{\prime \prime}, \quad\left[h_{1}, L_{ \pm}\right]= \pm L_{ \pm}, \\
{\left[h_{2}, L_{ \pm}\right] } & =\mp L_{ \pm}, \quad\left[b^{\prime}, b^{\prime \prime}\right]=h_{1}+h_{2}, \\
{\left[b^{\prime}, L_{-}\right] } & =2 a_{1}^{\prime}, \quad\left[b^{\prime}, L_{+}\right]=2 a_{2}^{\prime}, \quad\left[b^{\prime \prime}, L_{-}\right]=-2 a_{2}^{\prime \prime}, \\
{\left[b^{\prime \prime}, L_{+}\right] } & =-2 a_{1}^{\prime \prime}, \quad\left[a_{1}^{\prime}, b^{\prime \prime}\right]=\left[b^{\prime}, a_{2}^{\prime \prime}\right]=L_{-}, \\
{\left[a_{2}^{\prime}, b^{\prime \prime}\right] } & =\left[b^{\prime}, a_{1}^{\prime \prime}\right]=L_{+}, \quad\left[a_{1}^{\prime}, L_{+}\right]=\left[a_{2}^{\prime}, L_{-}\right]=b^{\prime}, \\
{\left[a_{2}^{\prime \prime}, L_{+}\right] } & =\left[a_{1}^{\prime \prime}, L_{-}\right]=-b^{\prime \prime} .
\end{aligned}
$$

На первый взгляд, эти соотношения могут показаться достаточно хаотичными, но фактически они совершенно естественны в вейлевском базисе алгебры $s o(2,3)$.

Приведенные два набора из десяти операторов связаны соотношениями

$$
\begin{aligned}
& M_{10}=i\left(a_{1}^{\prime \prime}-a_{1}^{\prime}-a_{2}^{\prime \prime}+a_{2}^{\prime}\right), \quad M_{15}=a_{2}^{\prime \prime}+a_{2}^{\prime}-a_{1}^{\prime \prime}-a_{1}^{\prime}, \\
& M_{20}=a_{1}^{\prime \prime}+a_{2}^{\prime \prime}+a_{1}^{\prime}+a_{2}^{\prime}, \quad M_{25}=i\left(a_{1}^{\prime \prime}+a_{2}^{\prime \prime}-a_{1}^{\prime}-a_{2}^{\prime}\right), \\
& M_{12}=L_{3}, \quad M_{23}=L_{+}+L_{-}, \quad M_{31}=-i\left(L_{+}-L_{-}\right), \\
& M_{05}=h_{1}+h_{2}, \quad M_{35}=b^{\prime}+b^{\prime \prime}, \quad M_{30}=-i\left(b^{\prime \prime}-b^{\prime}\right) .
\end{aligned}
$$

Кроме того, если $L_{+}^{*}=L_{-}, a_{j}^{\prime *}=a_{j}^{\prime \prime}, b^{*}=b^{\prime \prime}$ и $h_{j}^{*}=h_{j}$, где звездочка обозначает эрмитово сопряжение, то операторы $M^{a b}$ эрмитовы (мы не обсуждаем различие между эрмитовыми и самосопряженными операторами).

Пусть $p$ - простое число и $F_{p^{2}}$ - поле Галуа, содержащее $p^{2}$ элементов. Это поле имеет единственный нетривиальный автоморфизм (см., например, [4], [5]), который служит аналогом комплексного сопряжения в поле комплексных чисел. Этот автоморфизм можно определить как $a \rightarrow \bar{a}=a^{p}[5]$. Наша цель - найти НП, реализующие коммутационные соотношения (2)-(4) в пространствах над $F_{p^{2}}$. Представления в пространствах над полями ненулевой характеристики называются модулярными представлениями. Обзор теории модулярных НП можно найти, например, в работе [6]. В данной статье нам не понадобится обшая теория, поскольку рассматриваемые модулярные НП могут быть явно построены. Модулярным аналогом гильбертова пространства является линейное пространство $V$ над $F_{p^{2}}$, снабженное таким скалярным произведением $(\cdot, \cdot)$, что для любых $x, y \in V$ и $a \in F_{p^{2}},(x, y) \in F_{p^{2}}$ справедливы соотношения

$$
(x, y)=\overline{(y, x)}, \quad(a x, y)=\bar{a}(x, y), \quad(x, a y)=a(x, y) .
$$


В модулярном случае звездочка обозначает такое эрмитово сопряжение, что $(A x, y)=$ $\left(x, A^{*} y\right)$.

Формулы (2) определяют коммутационные соотношения для представлений алгебры $s p(2)$. Эти представления играют важную роль при построении модулярных НП алгебры $s o(2,3)$. Поэтому, следуя [3], [4], ниже мы описываем модулярные НП алгебры $s p(2)$, генераторы представления которых обозначаются через $a^{\prime}, a^{\prime \prime}, h$.

Оператор Казимира второго порядка для алгебры (2) имеет вид

$$
K=h^{2}-2 h-4 a^{\prime \prime} a^{\prime}=h^{2}+2 h-4 a^{\prime} a^{\prime \prime} .
$$

Мы рассмотрим представления с таким вектором $e_{0}$, что

$$
a^{\prime} e_{0}=0, \quad h e_{0}=q_{0} e_{0}, \quad\left(e_{0}, e_{0}\right)=1 .
$$

Нетрудно доказать [3], [4], что число $q_{0}$ является “вешественным", т.е. $q_{0} \in F_{p}$, где $F_{p}$ - поле вычетов по модулю $p: F_{p}=\mathbb{Z} / \mathbb{Z} p$ ( $\mathbb{Z}$ - кольцо целых чисел). Поле $F_{p}$ состоит из $p$ элементов и представляет собой простейшее из всех возможных полей Галуа.

Пусть $e_{n}=\left(a^{\prime \prime}\right)^{n} e_{0}$. Тогда из $(7)$ и (8) следует, что

$$
\begin{gathered}
h e_{n}=\left(q_{0}+2 n\right) e_{n}, \quad K e_{n}=q_{0}\left(q_{0}-2\right) e_{n}, \\
a^{\prime} a^{\prime \prime} e_{n}=(n+1)\left(q_{0}+n\right) e_{n}, \\
\left(e_{n+1}, e_{n+1}\right)=(n+1)\left(q_{0}+n\right)\left(e_{n}, e_{n}\right)
\end{gathered}
$$

для любых $n=0,1,2, \ldots$.

Так как случай $q_{0}=0$, соответствующий нулевому представлению, является тривиальным, то считаем, что $q_{0} \neq 0$. В этом случае обычные и модулярные представления значительно отличаются друг от друга. Рассмотрим сначала обычньй случай, когда $q_{0}$ - произвольное положительное вешественное число. Тогда НП бесконечномерно, $e_{0}$ - собственный вектор, отвечающий наименьшему собственному значению оператора $h$ (вектор минимального веса), и не сушествует векторов максимального веса. Это согласуется с тем известным фактом, что унитарные НП некомпактных групп бесконечномерны. В то же время в модулярном случае $q_{0}$ является одним из чисел $1, \ldots, p-1$. Множество $\left(e_{0}, e_{1}, \ldots, e_{N}\right)$ образует базис НП, если $a^{\prime \prime} e_{i} \neq 0$ при $i<N$ и $a^{\prime \prime} e_{N}=0$. Эти условия должны быть совместны с условием $a^{\prime} a^{\prime \prime} e_{N}=0$. Поэтому, как видно из (11), $N$ определяется условием $q_{0}+N=0$ в $F_{p}$. Следовательно, $N=p-q_{0}$, и размерность НП равна $p-q_{0}+1$.

Можно было бы считать, что $e_{0}$ является вектором минимального веса, а $e_{N}-$ вектором максимального веса. Однако понятия "меньше, чем" или “больше, чем" имеют ограниченную область применимости в случае $F_{p}$, так же как и понятие положительного и отрищательного числа в $F_{p}$. Если $q_{0}$ положительно в этом смысле (подробности 
см. в [4]), то, как показывают формулы (8) и (9), рассматриваемые модулярные НП могут рассматриваться как модулярный аналог НП с "положительной энергией". В то же время легко видеть, что $e_{N}$ является собственным вектором оператора $h$ с собственным значением $-q_{0}$ в $F_{p}$, и те же самые НП можно рассматривать как модулярный аналог НП с "отрицательной энергией" (подробности см. в [4]).

\section{3. БЕЗМАССОВЫЕ МОДУЛЯРНЫЕ ПРЕДСТАВЛЕНИЯ АЛГЕБРЫ AdS}

Имеется обширная литература по обычным НП алгебры $s o(2,3)$ в гильбертовом пространстве. Представления, отвечаюшие элементарным частицам в пространстве AdS, были впервые построены в работах [7], [8], а модулярные представления алгебры (2)-(4) впервые были изучены Браденом [9]. В [3], [4] мы переформулировали его результаты таким образом, чтобы получить прямое соответствие между модулярными и обычными НП. Наша конструкция приведена ниже.

Мы используем базис, в котором операторы $h_{j}, K_{j}, j=1,2$, диагональны. Здесь $K_{j}$ оператор Казимира (7) для алгебры $\left(a_{j}^{\prime}, a_{j}^{\prime \prime}, h_{j}\right)$. По аналогии с [8], [9] введем операторы

$$
\begin{aligned}
& B^{++}=b^{\prime \prime}-a_{1}^{\prime \prime} L_{-}\left(h_{1}-1\right)^{-1}-a_{2}^{\prime \prime} L_{+}\left(h_{2}-1\right)^{-1}+a_{1}^{\prime \prime} a_{2}^{\prime \prime} b^{\prime}\left[\left(h_{1}-1\right)\left(h_{2}-1\right)\right]^{-1} \\
& B^{+-}=L_{+}-a_{1}^{\prime \prime} b^{\prime}\left(h_{1}-1\right)^{-1}, \quad B^{-+}=L_{-}-a_{2}^{\prime \prime} b^{\prime}\left(h_{2}-1\right)^{-1}, \quad B^{--}=b^{\prime}
\end{aligned}
$$

и рассмотрим их действие лишь на пространстве "минимальных" $(s p(2) \times s p(2))$-векторов, т.е. векторов $x$ таких, что $a_{j}^{\prime} x=0$ при $j=1,2$ и $x$ является собственным вектором операторов $h_{j}$.

Легко видеть, что если $x$ является таким минимальным вектором, что $h_{j} x=\alpha_{j} x$, то $B^{++} x, B^{+-} x, B^{-+} x$ и $B^{--} x$ являются собственными векторами операторов $h_{j}$ с собственными значениями $\alpha_{j}+1,\left(\alpha_{1}+1, \alpha_{2}-1\right),\left(\alpha_{1}-1, \alpha_{2}+1\right)$ и $\alpha_{j}-1$, соответственно.

По аналогии с конструкцией обычных представлений с положительной энергией [7], [8] потребуем существования вектора $e_{0}$, удовлетворяющего условиям

$$
a_{j}^{\prime} e_{0}=b^{\prime} e_{0}=L_{+} e_{0}=0, \quad h_{j} e_{0}=q_{j} e_{0}, \quad\left(e_{0}, e_{0}\right)=1, \quad j=1,2 .
$$

В обычном случае безмассовые НП характеризуются условием $q_{2}=1$. В модулярном случае имеем то же условие, но теперь $q_{2} \in F_{p}$.

Хорошо известно, что $M^{05}=h_{1}+h_{2}$ является AdS-аналогом оператора энергии, так как $M^{05} / 2 R$ становится обычной энергией, когда группа AdS редуцируется до группы Пуанкаре (здесь $R$ - радиус пространства AdS). Из (2), (4) вытекает, что операторы $\left(a_{1}^{\prime}, a_{2}^{\prime}, b^{\prime}\right)$ уменьшают энергию $\mathrm{AdS}$ на две единицы. Таким образом, в обычной теории вектор $e_{0}$ является состоянием с наименьшей энергией. Спин в этой теории в наших единицах равен максимальному значению оператора $L_{3}=h_{1}-h_{2}$ в "состоянии покоя". Поэтому мы используем $s$ для обозначения $q_{1}-q_{2}$. В наших единицах $s=2$ для фотона 
и $s=4$ для гравитона. Отметим, что, в отличие от пуанкаре-инвариантных теорий, в случае AdS у безмассовых частиц есть состояния, которые можно рассматривать как состояния покоя (см. ниже).

Возникает вопрос, как определить действие операторов $B^{++}$и $B^{-+}$на вектор $e_{0}$, являющийся собственным вектором оператора $h_{2}$ с собственным значением $q_{2}=1$. Для раскрытия неопределенностей $0 / 0$ в матричных элементах можно записать $q_{2}$ в виде $q_{2}=1+\epsilon$ и перейти к пределу $\epsilon \rightarrow 0$ на завершаюшей стадии выгислений. Это подтверждает тот хорошо известный факт, что аналитические методы могут быть весьма полезны при решении задач, касающихся только целых чисел. В то же время полученные результаты можно воспроизвести, используя только целые числа (точнее, элементы рассматриваемого поля Галуа), но мы не будем на этом останавливаться подробнее.

Пользуясь указанной прескрипцией, потребуем, чтобы

$$
B^{++} e_{0}=\left[b^{\prime \prime}-a_{1}^{\prime \prime} L_{-}\left(h_{1}-1\right)^{-1}\right] e_{0}, \quad B^{-+} e_{0}=L_{-} e_{0}
$$

при $s \neq 0$ (и, следовательно, $h_{1} \neq 1$ ) и

$$
B^{++} e_{0}=b^{\prime \prime} e_{0}, \quad B^{+-} e_{0}=B^{-+} e_{0}=0
$$

при $s=0$. Из предыдуших замечаний следует, что определенные таким образом операторы переводят минимальные векторы в минимальные векторы. Следовательно, элемент

$$
e_{n k}=\left(B^{++}\right)^{n}\left(B^{-+}\right)^{k} e_{0}
$$

является минимальным $(s p(2) \times s p(2))$-вектором. Отвечающие ему собственные значения операторов $h_{1}$ и $h_{2}$ равны соответственно $q_{1}+n-k$ и $q_{2}+n+k$.

Прямая проверка показывает, что из (2)-(4) вытекают соотношения

$$
\begin{aligned}
& B^{-+} B^{++}\left(h_{1}-1\right)=B^{++} B^{-+}\left(h_{1}-2\right), \\
& B^{+-} B^{++}\left(h_{2}-1\right)=B^{++} B^{+-}\left(h_{2}-2\right) .
\end{aligned}
$$

Кроме того, из (13) получаем (подробности см. в [4])

$$
B^{--} e_{n k}=a(n, k) e_{n-1, k}, \quad B^{+-} e_{n k}=b(n, k) e_{n, k-1},
$$

где

$$
\begin{aligned}
& a(n, k)=\frac{n\left(q_{1}+q_{2}+n-3\right)\left(q_{1}+n-1\right)\left(q_{2}+n-2\right)}{\left(q_{1}+n-k-2\right)\left(q_{2}+n+k-2\right)}, \\
& b(n, k)=\frac{k(s+1-k)\left(q_{2}+k-2\right)}{q_{2}+n+k-2} .
\end{aligned}
$$

Из этих выражений следует, что элементы $e_{n k}$ образуют базис в пространстве минимальных $(s p(2) \times s p(2))$-векторов, и нашей следуюшей целью является определение области значений чисел $n$ и $k$. 
Рассмотрим величину $b(0, k)=k(s+1-k)$ и обозначим через $k_{\max }$ максимальное значение $k$. Если $k_{\max } \neq 0$, то для непротиворечивости нужно потребовать, чтобы $k=$ $k_{\max }$ было наибольшим значением $k$ таким, что $b(0, k) \neq 0$ при $k=1, \ldots, k_{\max }$. Отсюда заключаем, что $k$ может принимать только значения $0,1, \ldots, s$.

Пусть теперь $n_{\max }(k)$ - максимальное значение $n$ при заданном $k$. Если $n_{\max }(k) \neq 0$, то для непротиворечивости нужно потребовать, чтобы $n_{\max }(k)$ было наибольшим значением $n$ таким, что $a(n, k) \neq 0$ при $n=1, \ldots, n_{\max }(k)$. Из (19) вытекает, что в безмассовом случае (когда $\left.q_{2}=1\right) a(1, k)=0$ при $k=1, \ldots, s-1$, если имеются такие значения $k$ (т.е. когда $s \geqslant 2$ ), и $a(n, k)=n(s+n)$, если $k=0$ или $k=s$. Поэтому заключаем, что при $k=1, \ldots, s-1$ величина $n$ может принимать только значение $n=0$, в то время как при $k=0$ или $k=s$ возможными значениями $n$ являются $0,1, \ldots, n_{\max }$, где $n_{\max }=p-s-1$ (в отличие от стандартной теории, где $\left.n=0,1, \ldots, \infty\right)$.

Полный базис пространства представления можно выбрать в виде

$$
e\left(n_{1}, n_{2}, n, k\right)=\left(a_{1}^{\prime \prime}\right)^{n_{1}}\left(a_{2}^{\prime \prime}\right)^{n_{2}} e_{n k}
$$

где

$$
\begin{array}{cl}
n_{1}=0,1, \ldots, N_{1}(n, k), & n_{2}=0,1, \ldots, N_{2}(n, k), \\
N_{1}(n, k)=p-q_{1}-n+k, & N_{2}(n, k)=p-q_{2}-n-k,
\end{array}
$$

согласно результатам, полученным в предыдушем разделе.

Мы приходим к заключению, что, в отличие от стандартной теории, где НП алгебр Ли эрмитовыми операторами обязательно бесконечномерны, безмассовые модулярные НП являются конечномерными и даже конечными, поскольку поле $F_{p^{2}}$ конечно. Это согласуется с обшим утверждением, доказанным Зассенхаусом [10], что любое модулярное НП конечномерно.

Обсудим теперь, почему рассматриваемые НП можно считать безмассовыми. Легко видеть, что

$$
\begin{aligned}
h_{1} e\left(n_{1}, n_{2}, n, k\right) & =\left(q_{1}+n-k+2 n_{1}\right) e\left(n_{1}, n_{2}, n, k\right), \\
h_{2} e\left(n_{1}, n_{2}, n, k\right) & =\left(q_{2}+n+k+2 n_{2}\right) e\left(n_{1}, n_{2}, n, k\right), \\
M^{05} e\left(n_{1}, n_{2}, n, k\right) & =\left(q_{1}+q_{2}+2 n+2 n_{1}+2 n_{2}\right) e\left(n_{1}, n_{2}, n, k\right) .
\end{aligned}
$$

Поэтому в стандартной AdS-теории соответствуюшее НП характеризуется минимальной энергией $\mathrm{AdS}$, равной $q_{1}+q_{2}=2 q_{2}+s$. Так как в обычном случае масса рассматривается как минимальная энергия и обычная энергия равна $M^{05} / 2 R$, то обычная масса обрашается в нуль при $q_{2}=1$ и $R \rightarrow \infty$. Однако этого наблюдения недостаточно, чтобы считать значение $q_{2}=1$ выделенным среди других значений $q_{2}$, так как $\left(2 q_{2}+s\right) / 2 R \rightarrow 0$ при $R \rightarrow \infty$ для любого конечного числа $q_{2}$. Напомним, что безмассовые частицы в обычной теории не имеют "состояний покоя" и, следовательно, значение $s$ не характеризует число состояний в соответствуюшем представлении алгебры $s u(2)$. Вместо этого 
безмассовые частищы характеризуются спиральностью, принимающей только два значения: $s$ или $-s$. В случае AdS аналогом этой ситуации является то обстоятельство, что при $q_{2}=1$ и $n>0$ имеются лишь элементы $e_{n k}$ с $k=0$ и $k=s$. Только при $n=0$ сушествуют элементы $e_{n k}$ с $k=0,1, \ldots, s$. Когда алгебра AdS редуцируется до алгебры Пуанкаре (смысл такой редукции хорошо известен [11]), дискретный спектр становится непрерывным, а вероятность того, что частиша имеет нулевую энергию, становится пренебрежимо малой.

Ввиду приведенных выше замечаний безмассовый случай часто характеризуется в литературе не только условием $q_{2}=1$, но и условием $s \geqslant 2$, поскольку только в этом случае $1 \leqslant s-1$. Мы лишь предполагаем, что $q_{2}=1$, в то время как спин может быть произвольным.

Подытоживая полученные выше результаты, получаем

$$
\begin{aligned}
& B^{++} e_{n k}=e_{n+1, k}, \quad k=0, s, \quad n=0,1, \ldots, n_{\max }-1, \\
& B^{--} e_{n k}=n(s+n) e_{n-1, k}, \quad k=0, s, n=1, \ldots, n_{\max }, \\
& B^{+-} e_{0 k}=k(s+1-k) e_{0, k-1}, \quad k=1, \ldots, s, \\
& B^{-+} e_{0 k}=e_{0, k+1}, \quad k=0,1, \ldots, s-1,
\end{aligned}
$$

а при других значениях $n$ и $k$ действие этих операторов на $e_{n k}$ равно нулю.

Наша следуюшая задача состоит в вычислении величин

$$
\operatorname{Norm}\left(n_{1}, n_{2}, n, k\right)=\left(e\left(n_{1}, n_{2}, n, k\right), e\left(n_{1}, n_{2}, n, k\right)\right) .
$$

Пользуясь (11) и (23), можно показать, что

$$
\operatorname{Norm}\left(n_{1}, n_{2}, n, k\right)=F\left(n_{1}, n_{2}, n, k\right) G(k),
$$

где

$$
F\left(n_{1}, n_{2}, n, k\right)=n_{1} ! n_{2} !\left(n_{1}+n+s-k\right) !\left(n+n_{2}+k\right) !, \quad G(k)=\frac{s !}{[(s-k) !]^{2}}
$$

В стандартных пуанкаре- и AdS-теориях также имеются НП с отрицательной энергией (как было отмечено в разделе 1 , в стандартном подходе они не используются), которые могут быть построены по аналогии с НП с положительной энергией. Вместо условий (13) можно потребовать сушествования такого вектора $e_{0}^{\prime}$, что

$$
a_{j}^{\prime \prime} e_{0}^{\prime}=b^{\prime \prime} e_{0}^{\prime}=L_{-} e_{0}^{\prime}=0, \quad h_{j} e_{0}^{\prime}=-q_{j} e_{0}^{\prime}, \quad\left(e_{0}^{\prime}, e_{0}^{\prime}\right) \neq 0, \quad j=1,2,
$$

где величины $q_{1}, q_{2}$ те же самые, что и для НП с положительной энергией. Очевидно, что НП с положительной и отрицательной энергией полностью независимы, поскольку для 
таких НП спектр оператора $M^{05}$ является соответственно положительным или отрицательным. В то же время, как показано в [4], модулярный аналог НП с положительной әнергией, характеризующегося некоторыми $q_{1}, q_{2}$ в формуле (13), и модулярный аналог НП с отрицательной энергией, характеризующегося теми же значениями $q_{1}, q_{2}$ в формуле (25), представляют собой одно и то же модулярное НП. Так как в этом заключается ключевое различие между стандартной квантовой теорией и КТПГ, то ниже мы даем доказательство (слегка отличаюшееся от приведенного в [4]).

Пусть $e_{0}$ - вектор, удовлетворяюший соотношениям (13), $N_{1}=p-q_{1}$ и $N_{2}=p-q_{2}$. Докажем, что вектор $x=\left(a_{1}^{\prime \prime}\right)^{N_{1}}\left(a_{2}^{\prime \prime}\right)^{N_{2}} e_{0}$ удовлетворяет условиям (25), т.е. $x$ можно отождествить с $e_{0}^{\prime}$.

Из формулы (9), определения $N_{1}, N_{2}$ и результатов предыдушего раздела вытекает, что $x$ является собственным вектором операторов $h_{1}$ и $h_{2}$ с собственными значениями $-q_{1}$ и $-q_{2}$, соответственно, и, кроме того, удовлетворяет условиям $a_{1}^{\prime \prime} x=a_{2}^{\prime \prime} x=0$.

Докажем теперь, что $b^{\prime \prime} x=0$. Так как $b^{\prime \prime}$ коммутирует с $a_{j}^{\prime \prime}$, то $b^{\prime \prime} x$ можно записать в виде

$$
b^{\prime \prime} x=\left(a_{1}^{\prime \prime}\right)^{N_{1}}\left(a_{2}^{\prime \prime}\right)^{N_{2}} b^{\prime \prime} e_{0} .
$$

Из формул (4) и (13) следует, что $a_{2}^{\prime} b^{\prime \prime} e_{0}=L_{+} e_{0}=0$, а $b^{\prime \prime} e_{0}$ является собственным вектором оператора $h_{2}$ с собственным значением $q_{2}+1$. Следовательно, $b^{\prime \prime} e_{0}$ является минимальным вектором представления алгебры $s p(2)$ размерности $p-q_{2}=N_{2}$. Поэтому $\left(a_{2}^{\prime \prime}\right)^{N_{2}} b^{\prime \prime} e_{0}=0$ и $b^{\prime \prime} x=0$.

Следуюший шаг состоит в доказательстве того, что $L_{-} x=0$. Из (4) и определения $x$ следует, что

$$
L_{-} x=\left(a_{1}^{\prime \prime}\right)^{N_{1}}\left(a_{2}^{\prime \prime}\right)^{N_{2}} L_{-} e_{0}-N_{1}\left(a_{1}^{\prime \prime}\right)^{N_{1}-1}\left(a_{2}^{\prime \prime}\right)^{N_{2}} b^{\prime \prime} e_{0} .
$$

Мы уже показали, что $\left(a_{2}^{\prime \prime}\right)^{N_{2}} b^{\prime \prime} e_{0}=0$, и, следовательно, достаточно установить, что первый член в правой части (27) равен нулю. Из (4) и (13) вытекает, что $a_{2}^{\prime} L_{-} e_{0}=$ $b^{\prime} e_{0}=0$, a $L_{-} e_{0}$ является собственным вектором оператора $h_{2}$ с собственным значением $q_{2}+1$. Следовательно, $\left(a_{2}^{\prime \prime}\right)^{N_{2}} L_{-} e_{0}=0$ и соотношение $L_{-} x=0$ доказано.

То, что $(x, x) \neq 0$, непосредственно следует из определения вектора $x$ и результатов предыдушего раздела. Поэтому вектор $x$ действительно можно отождествить с $e_{0}^{\prime} . \mathrm{У}_{\text {т- }}$ верждение доказано.

Матричные элементы оператора $M^{a b}$ определяются соотношениями

$$
M^{a b} e\left(n_{1}, n_{2}, n, k\right)=\sum_{n_{1}^{\prime}, n_{2}^{\prime}, n^{\prime}, k^{\prime}} M^{a b}\left(n_{1}^{\prime}, n_{2}^{\prime}, n^{\prime}, k^{\prime} ; n_{1}, n_{2}, n, k\right) e\left(n_{1}^{\prime}, n_{2}^{\prime}, n^{\prime}, k^{\prime}\right) .
$$

В модулярном случае след каждого оператора $M^{a b}$ равен нулю. Для операторов $a_{j}^{\prime}$, $a_{j}^{\prime \prime}, L_{ \pm}, b^{\prime}, b^{\prime \prime}$ это непосредственно очевидно: поскольку они вообше не имеют ненулевых диагональных элементов, они обязательно меняют одно из квантовых чисел $n_{1}, n_{2}, n, k$. 
Докажем это утверждение для диагональных операторов $h_{1}$ и $h_{2}$. Для каждого НП алгебры $s p(2)$ минимального веса $q_{0}$ и размерности $N+1$ оператор $h$ имеет собственные значения $q_{0}, q_{0}+2, \ldots, q_{0}+2 N$. Их сумма равна нулю в $F_{p}$, так как $q_{0}+N=0$ в $F_{p}$ (см. предыдущий раздел). Поэтому заключаем, что

$$
\sum_{n_{1}, n_{2}, n, k} M^{a b}\left(n_{1}, n_{2}, n, k ; n_{1}, n_{2}, n, k\right)=0 .
$$

Это свойство очень важно для исследования новой симметрии между частицами и античастицами (см. раздел 6).

\section{4. НОВАЯ СИММЕТРИЯ МЕЖДУ ЧАСТИЦАМИ И АНТИЧАСТИЦАМИ В КТПГ}

Так как $\left(n_{1}, n_{2}, n, k\right)$ - полный набор квантовых чисел для рассматриваемой элементарной частицы, то можно определить операторы, описывающие уничтожение и рождение частицы в состояниях с такими квантовыми числами. Пусть $a\left(n_{1}, n_{2}, n, k\right)$ - оператор уничтожения частицы в состоянии, описываемом вектором $e\left(n_{1}, n_{2}, n, k\right)$. Тогда сопряженный оператор $a^{*}\left(n_{1}, n_{2}, n, k\right)$ имеет смысл оператора рождения частицы в этом состоянии. Поскольку мы не нормируем состояния $e\left(n_{1}, n_{2}, n, k\right)$ на единицу (см. обсуждение в [4]), потребуем, чтобы операторы $a\left(n_{1}, n_{2}, n, k\right)$ и $a^{*}\left(n_{1}, n_{2}, n, k\right)$ удовлетворяли либо антикоммутационным соотношениям

$$
\left\{a\left(n_{1}, n_{2}, n, k\right), a^{*}\left(n_{1}^{\prime}, n_{2}^{\prime}, n^{\prime}, k^{\prime}\right)\right\}=\operatorname{Norm}\left(n_{1}, n_{2}, n, k\right) \delta_{n_{1} n_{1}^{\prime}} \delta_{n_{2} n_{2}^{\prime}} \delta_{n n^{\prime}} \delta_{k k^{\prime}},
$$

либо коммутационным соотношениям

$$
\left[a\left(n_{1}, n_{2}, n, k\right), a^{*}\left(n_{1}^{\prime}, n_{2}^{\prime}, n^{\prime}, k^{\prime}\right)\right]=\operatorname{Norm}\left(n_{1}, n_{2}, n, k\right) \delta_{n_{1} n_{1}^{\prime}} \delta_{n_{2} n_{2}^{\prime}} \delta_{n n^{\prime}} \delta_{k k^{\prime}}
$$

Тогда, учитывая, что матричные элементы удовлетворяют правильным коммутационным соотношениям, легко показать, что операторы $M^{a b}$ во вторично-квантованной формe

$$
M^{a b}=\sum \frac{M^{a b}\left(n_{1}^{\prime}, n_{2}^{\prime}, n^{\prime}, k^{\prime} ; n_{1}, n_{2}, n, k\right) a^{*}\left(n_{1}^{\prime}, n_{2}^{\prime}, n^{\prime}, k^{\prime}\right) a\left(n_{1}, n_{2}, n, k\right)}{\operatorname{Norm}\left(n_{1}, n_{2}, n, k\right)}
$$

удовлетворяют коммутационным соотношениям вида (1) или (2)-(4), если операторы $a$, $a^{*}$ удовлетворяют условию (30) или (31). Здесь и ниже суммирование проводится по повторяющимся индексам.

В стандартной теории, где частица и ее античастица описываются независимыми НП, формула (32) описывает квантованное поле частицы или античастицы. Точнее, предположим, что операторы $a\left(n_{1}, n_{2}, n, k\right)$ и $a^{*}\left(n_{1}, n_{2}, n, k\right)$ относятся к частицам, а 
операторы $b\left(n_{1}, n_{2}, n, k\right)$ и $b^{*}\left(n_{1}, n_{2}, n, k\right)$ удовлетворяют аналогичным коммутационным соотношениям и описывают уничтожение и рождение античастиц. Тогда в стандартной теории операторы квантованного поля частищ и античастиц имеют вид

$$
\begin{aligned}
M_{\text {standard }}^{a b} & =\sum \frac{M_{\text {particle }}^{a b}\left(n_{1}^{\prime}, n_{2}^{\prime}, n^{\prime}, k^{\prime} ; n_{1}, n_{2}, n, k\right) a^{*}\left(n_{1}^{\prime}, n_{2}^{\prime}, n^{\prime}, k^{\prime}\right) a\left(n_{1}, n_{2}, n, k\right)}{\operatorname{Norm}\left(n_{1}, n_{2}, n, k\right)}+ \\
+ & \sum \frac{M_{\text {antiparticle }}^{a b}\left(n_{1}^{\prime}, n_{2}^{\prime}, n^{\prime}, k^{\prime} ; n_{1}, n_{2}, n, k\right) b^{*}\left(n_{1}^{\prime}, n_{2}^{\prime}, n^{\prime}, k^{\prime}\right) b\left(n_{1}, n_{2}, n, k\right)}{\operatorname{Norm}\left(n_{1}, n_{2}, n, k\right)},
\end{aligned}
$$

где квантовые числа $\left(n_{1}, n_{2}, n, k\right)$ в каждой сумме принимают значения, допустимые для соответствуюшего НП

В отличие от стандартной теории, формула (32) описывает квантованное поле одновременно для частиц и античастиц. Если значения $\left(n_{1}, n_{2}, n\right)$ много меньше $p$, то вклад таких значений правильно описьвает частицы (подробности см. в [4]). Возникает вопрос о том, дает ли это выражение правильное описание вклада античастиц в КТПГ. В самом деле, если энергия $\mathrm{AdS}$ отрицательна, то оператор $a\left(n_{1}, n_{2}, n, k\right)$ нельзя рассматривать как оператор уничтожения, а $a^{*}\left(n_{1}, n_{2}, n, k\right)$ нельзя рассматривать как оператор рождения.

Напомним (см. раздел 3$)$, что при произвольных фиксированных значениях $n$ и $k$ величины $n_{1}$ и $n_{2}$ могут принимать только значения $0,1, \ldots, N_{1}(n, k)$ и $0,1, \ldots, N_{2}(n, k)$, соответственно (см. (21)). Обозначим $q_{1}+n-k$ и $q_{2}+n+k$ через $Q_{1}(n, k)$ и $Q_{2}(n, k)$, соответственно. Из $(22)$ следует, что элемент $e\left(n_{1}, n_{2}, n, k\right)$ является собственным вектором операторов $h_{1}$ и $h_{2}$ с собственными значениями $Q_{1}(n, k)+2 n_{1}$ и $Q_{2}(n, k)+2 n_{2}$, соответственно. Из результатов раздела 2 вытекает, что первое НП алгебры $s p(2)$ имеет размерность $N_{1}(n, k)+1$, а второе НП имеет размерность $N_{2}(n, k)+1$. Если $n_{1}=N_{1}(n, k)$, то ввиду (22) первое собственное значение равно $-Q_{1}(n, k)$ в $F_{p}$, а если $n_{2}=N_{2}(n, k)$, то второе собственное значение равно $-Q_{2}(n, k)$ в $F_{p}$. Обозначим $N_{1}(n, k)-n_{1}$ и $N_{2}(n, k)-$ $n_{2}$ через $\tilde{n}_{1}$ и $\tilde{n}_{2}$, соответственно. Из $(22)$ вытекает, что $e\left(\tilde{n}_{1}, \tilde{n}_{2}, n, k\right)$ является собственным вектором оператора $h_{1}$ с собственным значением $-\left(Q_{1}(n, k)+2 n_{1}\right)$ и собственным вектором оператора $h_{2}$ с собственным значением $-\left(Q_{2}(n, k)+2 n_{2}\right)$.

В КТПГ операторы $b\left(n_{1}, n_{2}, n, k\right)$ и $b^{*}\left(n_{1}, n_{2}, n, k\right)$ не могут быть независимыми от $a\left(n_{1}, n_{2}, n, k\right)$ и $a^{*}\left(n_{1}, n_{2}, n, k\right)$. По своему смыслу операторы $b\left(n_{1}, n_{2}, n, k\right)$ и $b^{*}\left(n_{1}, n_{2}, n, k\right)$ должны быть такими, чтобы их можно было интерпретировать как операторы уничтожения и рождения античастиц при условии, что значения $\left(n_{1}, n_{2}, n\right)$ много меньше $p$. Поэтому разумно предположить, что оператор $b\left(n_{1}, n_{2}, n, k\right)$ следует определить так, чтобы он был пропорционален $a^{*}\left(\tilde{n}_{1}, \tilde{n}_{2}, n, k\right)$, а $b^{*}\left(n_{1}, n_{2}, n, k\right)$ следует определить так, чтобы он был пропорционален $a\left(\tilde{n}_{1}, \tilde{n}_{2}, n, k\right)$. Таким образом, можно прямо реализовать идею о том, что рождение античастицы с положительной энергией может быть описано как уничтожение частицы с отрицательной энергией, а уничтожение античастицы с положительной энергией может быть описано как рождение частицы с отрицательной энергией. Как было отмечено в разделе 1, в стандартной теории эта идея реализуется неявно. 
Так как $(p-1) !=-1$ в $F_{p}$ в силу известной теоремы Вильсона (см., например, [5]), то из (24) следует, что

$$
F\left(n_{1}, n_{2}, n, k\right) F\left(\tilde{n}_{1}, \tilde{n}_{2}, n, k\right)=(-1)^{s}
$$

Определим теперь $b$-операторы следующим образом:

$$
a^{*}\left(n_{1}, n_{2}, n, k\right)=\frac{\eta\left(n_{1}, n_{2}, n, k\right) b\left(\tilde{n}_{1}, \tilde{n}_{2}, n, k\right)}{F\left(\tilde{n}_{1}, \tilde{n}_{2}, n, k\right)},
$$

где $\eta\left(n_{1}, n_{2}, n, k\right)$ - некоторая функция. Отметим, что в стандартной теории $C P T$-преобразование в формулировке Швингера переводит $a^{*}$ в $b$ (см., например, [12], [13]), причем, в отличие от (35), оба оператора рассматриваются при положительных энергиях. В отличие от стандартного $C P T$-преобразования, где наборы $\left(a, a^{*}\right)$ и $\left(b, b^{*}\right)$ независимы, соотношение (35) представляет собой не преобразование, а определение.

Из этого определения следует, что

$$
\begin{aligned}
a\left(n_{1}, n_{2}, n, k\right) & =\frac{\bar{\eta}\left(n_{1}, n_{2}, n, k\right) b^{*}\left(\tilde{n}_{1}, \tilde{n}_{2}, n, k\right)}{F\left(\tilde{n}_{1}, \tilde{n}_{2}, n, k\right)}, \\
b^{*}\left(n_{1}, n_{2}, n, k\right) & =\frac{a\left(\tilde{n}_{1}, \tilde{n}_{2}, n, k\right) F\left(n_{1}, n_{2}, n, k\right)}{\bar{\eta}\left(\tilde{n}_{1}, \tilde{n}_{2}, n, k\right)}, \\
b\left(n_{1}, n_{2}, n, k\right) & =\frac{a^{*}\left(\tilde{n}_{1}, \tilde{n}_{2}, n, k\right) F\left(n_{1}, n_{2}, n, k\right)}{\eta\left(\tilde{n}_{1}, \tilde{n}_{2}, n, k\right)} .
\end{aligned}
$$

Формулы (35) и (36) определяют возможную симметрию, если набор $\left(a, a^{*}\right)$ заменить на набор $\left(b, b^{*}\right)$. Назовем ее $A B$-симметрией. Чтобы понять, действительно ли это новая симметрия, нам надо исследовать, при каких условиях определенные таким образом операторы $\left(b, b^{*}\right)$ удовлетворяют тем же коммутационным или антикоммутационным соотношениям, что и операторы $\left(a, a^{*}\right)$, и выяснить, имеют ли операторы $M^{a b}$, записанные в терминах $\left(b, b^{*}\right)$ и $\left(a, a^{*}\right)$, одинаковый вид.

Из (30) и (31) следует, что $b$-операторы должны удовлетворять соотношению

$$
\left\{b\left(n_{1}, n_{2}, n, k\right), b^{*}\left(n_{1}^{\prime}, n_{2}^{\prime}, n^{\prime}, k^{\prime}\right)\right\}=\operatorname{Norm}\left(n_{1}, n_{2}, n, k\right) \delta_{n_{1} n_{1}^{\prime}} \delta_{n_{2} n_{2}^{\prime}} \delta_{n n^{\prime}} \delta_{k k^{\prime}}
$$

в случае антикоммутаторов и соотношению

$$
\left[b\left(n_{1}, n_{2}, n, k\right), b^{*}\left(n_{1}^{\prime}, n_{2}^{\prime}, n^{\prime}, k^{\prime}\right)\right]=\operatorname{Norm}\left(n_{1}, n_{2}, n, k\right) \delta_{n_{1} n_{1}^{\prime}} \delta_{n_{2} n_{2}^{\prime}} \delta_{n n^{\prime}} \delta_{k k^{\prime}}
$$

в случае коммутаторов.

Теперь из (24), (30), (34)-(36) вытекает, что соотношение (37) выполнено, если

$$
\eta\left(n_{1}, n_{2}, n, k\right) \bar{\eta}\left(n_{1}, n_{2}, n, k\right)=(-1)^{s} .
$$


В то же время в случае коммутаторов из (24), (31) и (34)-(36) следует, что соотношение (38) выполнено, если

$$
\eta\left(n_{1}, n_{2}, n, k\right) \bar{\eta}\left(n_{1}, n_{2}, n, k\right)=(-1)^{s+1} .
$$

Представим теперь $\eta\left(n_{1}, n_{2}, n, k\right)$ в виде

$$
\eta\left(n_{1}, n_{2}, n, k\right)=\alpha f\left(n_{1}, n_{2}, n, k\right)
$$

где $f\left(n_{1}, n_{2}, n, k\right)$ удовлетворяет условию

$$
f\left(n_{1}, n_{2}, n, k\right) \bar{f}\left(n_{1}, n_{2}, n, k\right)=1 .
$$

Тогда $\alpha$ должно удовлетворять соотношению

$$
\alpha \bar{\alpha}= \pm(-1)^{s}
$$

где знак плюс относится к антикоммутаторам, а знак минус - к коммутаторам. Если справедлива теорема о связи спина со статистикой, т.е. антикоммутаторы соответствуют нечетным, а коммутаторы - четным значениям $s$ (в этом состоит содержание известной теоремы Паули локальной квантовой теории поля [14]), то правая часть (43) равна -1 .

Соотношение (43) следует из того, что наш базис не нормирован (обсуждение этого вопроса см. в [4]). В стандартной теории такое соотношение невозможно, но если $\alpha \in F_{p^{2}}$, то сушествует решение уравнения (43). Действительно, можно воспользоваться тем фактом, что поле Галуа циклично по отношению к умножению [5]. Пусть $r-$ примитивный корень $F_{p^{2}}$. Это означает, что каждый элемент $F_{p^{2}}$ представляет собой степень $r$. Как уже упоминалось в разделе 2 , имеется единственный автоморфизм $F_{p^{2}}$, определенный соотношением $\alpha \rightarrow \bar{\alpha}=\alpha^{p}$. Поэтому если $\alpha=r^{k}$, то $\alpha \bar{\alpha}=r^{(p+1) k}$. С другой стороны, так как $r^{\left(p^{2}-1\right)}=1$, то $r^{\left(p^{2}-1\right) / 2}=-1$. Следовательно, сушествует, по крайней мере, решение с $k=(p-1) / 2$.

\section{5. УСЛОВИЕ СУЩЕСТВОВАНИЯ ВАКУУМА}

Хотя мы и назвали наборы $\left(a, a^{*}\right)$ и $\left(b, b^{*}\right)$ операторами уничтожения и рождения соответственно частиц и античастиц, все же не очевидно, что их можно интерпретировать таким образом. В стандартном подходе в этом можно убедиться, используя следующую процедуру. Потребуем сушествования вакуумного вектора $\Phi_{0}$, удовлетворяющего условию

$$
a\left(n_{1}, n_{2}, n, k\right) \Phi_{0}=b\left(n_{1}, n_{2}, n, k\right) \Phi_{0}=0 \quad \forall n_{1}, n_{2}, n, k .
$$


Тогда элементы

$$
\Phi_{+}\left(n_{1}, n_{2}, n, k\right)=a^{*}\left(n_{1}, n_{2}, n, k\right) \Phi_{0}, \quad \Phi_{-}\left(n_{1}, n_{2}, n, k\right)=b^{*}\left(n_{1}, n_{2}, n, k\right) \Phi_{0}
$$

имеют смысл одночастичных состояний для частиц и античастиц, соответственно.

Однако если потребовать выполнения условия (44) в КТПГ, то ввиду (35) и (36) очевидно, что элементы, определенные при помощи (45), являются нулевыми векторами. Отметим, что в стандартном подходе энергия AdS всегда больше, чем масса, в то время как в KTПГ энергия AdS не является положительно-определенной. Поэтому попытаемся модифицировать условия (44) следующим образом. Прежде всего разобьем набор элементов $\left(n_{1}, n_{2}, n, k\right)$ на такие две равные непересекающиеся части $S_{+}$и $S_{-}$(которые будут определены позднее), что если $\left(n_{1}, n_{2}, n, k\right) \in S_{+}$, то $\left(\tilde{n}_{1}, \tilde{n}_{2}, n, k\right) \in S_{-}$. Затем вместо условия (44) наложим требование

$$
a\left(n_{1}, n_{2}, n, k\right) \Phi_{0}=b\left(n_{1}, n_{2}, n, k\right) \Phi_{0}=0 \quad \forall\left(n_{1}, n_{2}, n, k\right) \in S_{+} .
$$

Тогда элементы, определенные при помощи (45), действительно будут иметь смысл одночастичных состояний для $\left(n_{1}, n_{2}, n, k\right) \in S_{+}$.

По аналогии со стандартным подходом можно попытаться определить набор $S_{+}$таким образом, чтобы для соответствующих значений $\left(n_{1}, n_{2}, n, k\right)$ энергия $\mathrm{AdS} E=s+$ $2\left(n+n_{1}+n_{2}+1\right)$ была положительна. Однако, как уже отмечалось, положительность и отрицательность не имеют ясного смысла в $F_{p}$. Можно считать энергию AdS положительной, если все величины $\left(n, n_{1}, n_{2}\right)$ много меньше $p$, но в других случаях это неочевидно. По нашему мнению, в современной физике все еше нет полного понимания того, насколько важна положительность энергии. Поэтому ограничимся тем, что построим набор $S_{+}$математически непротиворечивым образом.

Будем говорить, что энергия AdS $E$ положительна (отрицательна), если $E$ равна одному из значений $1,2, \ldots,(p-1) / 2(-1,-2, \ldots,-(p-1) / 2)$. Если $E$ положительна (отрицательна), то потребуем, чтобы соответствующий элемент $\left(n_{1}, n_{2}, n, k\right)$ принадлежал $S_{+}\left(S_{-}\right)$. Проблема возникает с теми элементами, для которых соответствующее значение $E$ равно нулю в $F_{p}$. Напомним, что $E$ является собственным значением $M^{05}=h_{1}+h_{2}$, причем собственное значение $h_{1}$ равно $E^{(1)}=1+s+n-k+2 n_{1}$, а собственное значение $h_{2}$ равно $E^{(2)}=1+n+k+2 n_{2}$. Значение $E$ может быть равно нулю в трех случаях: 1) если $E^{(1)}$ положительно, а $E^{(2)}$ отрицательно; 2) если $E^{(1)}$ отрицательно, а $E^{(2)}$ положительно; 3) если $E^{(1)}=E^{(2)}=0$. Мы можем потребовать, чтобы соответствующий элемент $\left(n_{1}, n_{2}, n, k\right)$ в первом случае принадлежал $S_{+}$, а во втором случае принадлежал $S_{-}$. В то же время третий случай остается неясным.

Из результатов разделов 2 и 3 вытекает, что случай $E^{(1)}=0$ возможен лишь при $\tilde{n}_{1}=$ $n_{1}$, где $\tilde{n}_{1}=N_{1}(n, k)-n_{1}$, а $N_{1}(n, k)$ определено в $(21)$. Аналогично случай $E^{(2)}=0$ возможен лишш при $\tilde{n}_{2}=n_{2}$, где $\tilde{n}_{2}=N_{2}(n, k)-n_{2}$. Поэтому случай $E^{(1)}=0$ возможен, 
только если $N_{1}(n, k)$ четно и $n_{1}=N_{1}(n, k) / 2$. Аналогично случай $E^{(2)}=0$ возможен, только если $N_{2}(n, k)$ четно и $n_{2}=N_{2}(n, k) / 2$.

Теперь ясно, что если реализуется третий случай, то вся конструкция становится противоречивой. В самом деле, оператор $b\left(n_{1}, n_{2}, n, k\right)$ пропорционален $a^{*}\left(\tilde{n}_{1}, \tilde{n}_{2}, n, k\right)$. Поэтому если $\tilde{n}_{1}=n_{1}, \tilde{n}_{2}=n_{2}$ и вектор $\Phi_{0}$ аннигилируется как $a\left(n_{1}, n_{2}, n, k\right)$, так и $b\left(n_{1}, n_{2}, n, k\right)$, то он также аннигилируется обоими операторами $a\left(n_{1}, n_{2}, n, k\right)$ и $a^{*}\left(n_{1}, n_{2}, n, k\right)$. Но это противоречит соотношениям (30) и (31).

Так как $q_{1}=1+s$ и $q_{2}=1$ в безмассовом случае, то из (21) следует, что если $s$ четно, то $N_{1}(n, k)$ и $N_{2}(n, k)$ являются одновременно либо четными, либо нечетными. Поэтому в данном случае обязательно найдутся такие $(n, k)$, что обе величины $N_{1}(n, k)$ и $N_{2}(n, k)$ являются четными. Тогда при $n_{1}=N_{1}(n, k) / 2$ и $n_{1}=N_{1}(n, k) / 2$ с необходимостью имеем $E^{(1)}=E^{(2)}=0$. Более того, так как для каждого $(n, k)$ число всех возможных значений $\left(n_{1}, n_{2}, n, k\right)$ равно $\left(N_{1}(n, k)+1\right)\left(N_{2}(n, k)+1\right)$, то это число нечетно (и, следовательно, нельзя разделить множество всех возможных значений на две непересекаюшиеся части $S_{+}$и $\left.S_{-}\right)$.

С другой стороны, если $s$ нечетно, то для всех значений $(n, k)$ либо $N_{1}(n, k)$ четно, а $N_{2}(n, k)$ нечетно, либо $N_{1}(n, k)$ нечетно, а $N_{2}(n, k)$ четно. Следовательно, для любого значения $(n, k)$ случай $E^{(1)}=E^{(2)}=0$ невозможен, а число всех возможных значений $\left(n_{1}, n_{2}, n, k\right)$ четно.

Таким образом, мы приходим к заключению, что условие (46) математически непротиворечиво, только если $s$ нечетно или, другими словами, если спин частицы в обычных единицах является полуцелым.

Хотя интерпретация каждого оператора из набора $\left(a, a^{*}, b, b^{*}\right)$ как оператора рождения или уничтожения зависит от способа разбиения элементов $\left(n_{1}, n_{2}, n, k\right)$ на части $S_{+}$и $S_{-}$, требование непротиворечивости, приводящее к исключению случая $E^{(1)}=$ $E^{(2)}=0$, не зависит от выбора $S_{+}$и $S_{-}$. Поэтому, по нашему мнению, результаты данного раздела ясно указывают на то, что в безмассовом случае лишь частицы с полуцелым спином могут быть элементарными. Тогда из теоремы о связи спина со статистикой [14] следует, что безмассовые частищы могут быть описаны при помощи только антикоммутационных соотношений, т.е. они могут быть только фермионами. Однако в КТПГ теорема о связи спина со статистикой еше не доказана, поэтому в следующих разделах рассматриваются как антикоммутаторы, так и коммутаторы.

\section{6. СОГЛАСОВАННОСТЬ АВ-СИММЕТРИИ С ОПЕРАТОРАМИ ПРЕДСТАВЛЕНИЯ}

Рассмотрим операторы (32) и воспользуемся тем, что в модулярном случае след операторов $M^{a b}$ равен нулю (см. (29)). Тогда с учетом (30) и (31) соотношение (32) можно переписать в виде

$$
M^{a b}=\mp \sum \frac{M^{a b}\left(n_{1}^{\prime}, n_{2}^{\prime}, n^{\prime}, k^{\prime} ; n_{1}, n_{2}, n, k\right) a\left(n_{1}, n_{2}, n, k\right) a^{*}\left(n_{1}^{\prime}, n_{2}^{\prime}, n^{\prime}, k^{\prime}\right)}{\operatorname{Norm}\left(n_{1}, n_{2}, n, k\right)},
$$


где знак минус относится к антикоммутаторам, а знак плюс - к коммутаторам. Пользуясь (34)-(36) и (41)-(43), в обоих случаях получаем

$$
\begin{aligned}
M^{a b}=- & \sum \frac{M^{a b}\left(n_{1}^{\prime}, n_{2}^{\prime}, n^{\prime}, k^{\prime} ; n_{1}, n_{2}, n, k\right)}{\left[F\left(\tilde{n}_{1}^{\prime}, \tilde{n}_{2}^{\prime}, n^{\prime}, k^{\prime}\right) G(k)\right]} \times \\
& \times f\left(n_{1}^{\prime}, n_{2}^{\prime}, n^{\prime}, k^{\prime}\right) \bar{f}\left(n_{1}, n_{2}, n, k\right) b^{*}\left(\tilde{n}_{1}, \tilde{n}_{2}, n, k\right) b\left(\tilde{n}_{1}^{\prime}, \tilde{n}_{2}^{\prime}, n^{\prime}, k^{\prime}\right)= \\
= & -\sum \frac{M^{a b}\left(\tilde{n}_{1}, \tilde{n}_{2}, n, k ; \tilde{n}_{1}^{\prime}, \tilde{n}_{2}^{\prime}, n^{\prime}, k^{\prime}\right)}{\left[F\left(n_{1}, n_{2}, n, k\right) G\left(k^{\prime}\right)\right]} \times \\
& \times f\left(\tilde{n}_{1}, \tilde{n}_{2}, n, k\right) \bar{f}\left(\tilde{n}_{1}^{\prime}, \tilde{n}_{2}^{\prime}, n^{\prime}, k^{\prime}\right) b^{*}\left(n_{1}^{\prime}, n_{2}^{\prime}, n^{\prime}, k^{\prime}\right) b\left(n_{1}, n_{2}, n, k\right) .
\end{aligned}
$$

Рассмотрим сначала диагональный оператор энергии AdS. Из (22) следует, что в безмассовом случае матричные элементы оператора $M^{05}$ имеют вид

$$
M^{05}\left(n_{1}^{\prime}, n_{2}^{\prime}, n^{\prime}, k^{\prime} ; n_{1}, n_{2}, n, k\right)=\left(2+s+2 n+2 n_{1}+2 n_{2}\right) \delta_{n_{1} n_{1}^{\prime}} \delta_{n_{2} n_{2}^{\prime}} \delta_{n n^{\prime}} \delta_{k k^{\prime}}
$$

Следовательно, оператор (32) в данном случае дается выражением

$$
M^{05}=\sum_{n_{1}, n_{2}, n, k} \frac{\left[s+2\left(n+n_{1}+n_{2}+1\right)\right] a^{*}\left(n_{1}, n_{2}, n, k\right) a\left(n_{1}, n_{2}, n, k\right)}{\operatorname{Norm}\left(n_{1}, n_{2}, n, k\right)}
$$

В то же время из (42), (48), (49) и определения преобразования $n_{1} \rightarrow \tilde{n}_{1}, n_{2} \rightarrow \tilde{n}_{2}($ см. раздел 4) следует, что

$$
M^{05}=\sum_{n_{1}, n_{2}, n, k} \frac{\left[s+2\left(n+n_{1}+n_{2}+1\right)\right] b^{*}\left(n_{1}, n_{2}, n, k\right) b\left(n_{1}, n_{2}, n, k\right)}{\operatorname{Norm}\left(n_{1}, n_{2}, n, k\right)} .
$$

Суммы в $(50)$ и (51) берутся по всем значениям $\left(n_{1}, n_{2}, n, k\right)$, относяшимся к модулярному НП частицы. Вместе с тем, чтобы было соответствие со стандартным случаем, надо рассматривать только значения $\left(n_{1}, n_{2}, n\right)$, много меньшие $p$ (см. [3], [4]). Вывод формулы (51) показывает, что вклад таких $\left(n_{1}, n_{2}, n\right)$ происходит от вклада в $(50)$ тех $\left(n_{1}, n_{2}\right)$, для которых $\left(\tilde{n}_{1}, \tilde{n}_{2}\right)$ много меньше $p$. В этом случае значения $\left(n_{1}, n_{2}\right)$ сопоставимы с $p$. Поэтому, если рассматривать только те состояния, для которых $\left(n_{1}, n_{2}, n\right)$ в операторах $a$ и $b$ много меньше $p$, то гамильтониан $\mathrm{AdS}$ можно записать в виде

$$
\begin{aligned}
M^{05}= & \sum_{n_{1}, n_{2}, n, k}^{\prime} \frac{\left[s+2\left(n+n_{1}+n_{2}+1\right)\right]}{\operatorname{Norm}\left(n_{1}, n_{2}, n, k\right)} \times \\
& \times\left[a^{*}\left(n_{1}, n_{2}, n, k\right) a\left(n_{1}, n_{2}, n, k\right)+b^{*}\left(n_{1}, n_{2}, n, k\right) b\left(n_{1}, n_{2}, n, k\right)\right],
\end{aligned}
$$


где $\sum_{n_{1}, n_{2}, n, k}^{\prime}$ означает, что сумма берется только по тем значениям $\left(n_{1}, n_{2}, n, k\right)$, которые много меньше $p$. В этом выражении вклады частиц и античастиц явно выписаны, а соответствуюший гамильтониан $\mathrm{AdS}$ является положительно-определенным.

Полученные результаты показывают, что по отношению к оператору $M^{05}$ формула (35) действительно определяет новую симметрию, поскольку $M^{05}$ имеет один и тот же вид в терминах $\left(a, a^{*}\right)$ и $\left(b, b^{*}\right)(c p .(50)$ и (51)). Отметим, что мы не предполагали $C$-инвариантности теории (в стандартной теории $C$-инвариантность определяется как преобразование $\left.a\left(n_{1}, n_{2}, n, k\right) \leftrightarrow b\left(n_{1}, n_{2}, n, k\right)\right)$. Хорошо известно, что $C$-инвариантность не является фундаментальной симметрией. В стандартной теории фундаментальной является только $C P T$-инвариантность, так как согласно знаменитой $C P T$-теореме $[15]$ всякая локальная пуанкаре-инвариантная теория автоматически является $C P T$-инвариантной. Наше предположение заключается в том, что соотношение (35) определяет фундаментальную симметрию в КТПГ. Чтобы понять ее свойства, необходимо исследовать не только $M^{05}$, но и другие генераторы представления.

По аналогии со случаем оператора $M^{05}$ нетрудно показать, что при тех же условиях операторы $h_{1}$ и $h_{2}$ имеют одинаковый вид в терминах $\left(a, a^{*}\right)$ и $\left(b, b^{*}\right)$.

Рассмотрим теперь оператор $a_{1}^{\prime \prime}$ (см. раздел 2$)$. Из определения этого оператора следует, что его матричные элементы имеют вид

$$
a_{1}^{\prime \prime}\left(n_{1}^{\prime}, n_{2}^{\prime}, n^{\prime}, k^{\prime} ; n_{1}, n_{2}, n, k\right)=\delta_{n_{1}, n_{1}^{\prime}-1} \delta_{n_{2} n_{2}^{\prime}} \delta_{n n^{\prime}} \delta_{k k^{\prime}}
$$

и, следовательно, ввиду (32) во вторично-квантованной форме $a_{1}^{\prime \prime}$ дается выражением

$$
a_{1}^{\prime \prime}=\sum_{n_{1}=0}^{N_{1}-1} \sum_{n_{2}, n, k} \frac{a^{*}\left(n_{1}+1, n_{2}, n, k\right) a\left(n_{1}, n_{2}, n, k\right)}{\operatorname{Norm}\left(n_{1}, n_{2}, n, k\right)} .
$$

Нам надо доказать, что в терминах $\left(b, b^{*}\right)$ этот оператор имеет тот же самый вид, т.е. что также справедливо выражение

$$
a_{1}^{\prime \prime}=\sum_{n_{1}=0}^{N_{1}-1} \sum_{n_{2}, n, k} \frac{b^{*}\left(n_{1}+1, n_{2}, n, k\right) b\left(n_{1}, n_{2}, n, k\right)}{\operatorname{Norm}\left(n_{1}, n_{2}, n, k\right)} .
$$

Из (48) и (53) вытекает, что условие (55) действительно выполнено, если

$$
f\left(n_{1}, n_{2}, n, k\right) \bar{f}\left(n_{1}-1, n_{2}, n, k\right)=-1 .
$$

Из выражения

$$
a_{1}^{\prime} e\left(n_{1}, n_{2}, n, k\right)=a_{1}^{\prime} a_{1}^{\prime \prime} e\left(n_{1}-1, n_{2}, n, k\right)
$$

для действия оператора $a_{1}^{\prime}$ и формулы (10) следует, что матричные элементы оператора $a_{1}^{\prime}$ имеют вид

$$
a_{1}^{\prime}\left(n_{1}^{\prime}, n_{2}^{\prime}, n^{\prime}, k^{\prime} ; n_{1}, n_{2}, n, k\right)=n_{1}\left(Q_{1}(n, k)+n_{1}-1\right) \delta_{n_{1}, n_{1}^{\prime}+1} \delta_{n_{2} n_{2}^{\prime}} \delta_{n n^{\prime}} \delta_{k k^{\prime}}
$$


Поэтому, как видно из (32), этот оператор имеет следующую вторично-квантованную форому:

$$
a_{1}^{\prime}=\sum_{n_{1}=1}^{N_{1}} \sum_{n_{2}, n, k} \frac{n_{1}\left(Q_{1}(n, k)+n_{1}-1\right) a^{*}\left(n_{1}-1, n_{2}, n, k\right) a\left(n_{1}, n_{2}, n, k\right)}{\operatorname{Norm}\left(n_{1}, n_{2}, n, k\right)} .
$$

По аналогии с доказательством соотношения (55) можно показать, что в терминах $\left(b, b^{*}\right)$ этот оператор имеет тот же самый вид, т.е. что

$$
a_{1}^{\prime}=\sum_{n_{1}=1}^{N_{1}} \sum_{n_{2}, n, k} \frac{n_{1}\left(Q_{1}(n, k)+n_{1}-1\right) b^{*}\left(n_{1}-1, n_{2}, n, k\right) b\left(n_{1}, n_{2}, n, k\right)}{\operatorname{Norm}\left(n_{1}, n_{2}, n, k\right)}
$$

если

$$
f\left(n_{1}, n_{2}, n, k\right) \bar{f}\left(n_{1}+1, n_{2}, n, k\right)=-1 .
$$

Аналогичным образом можно доказать, что вторично-квантованные операторы $a_{2}^{\prime \prime}$ и $a_{2}^{\prime}$ также имеют одинаковый вид в терминах $\left(a, a^{*}\right)$ и $\left(b, b^{*}\right)$, если

$$
\begin{aligned}
& f\left(n_{1}, n_{2}, n, k\right) \bar{f}\left(n_{1}, n_{2}+1, n, k\right)=-1 \\
& f\left(n_{1}, n_{2}, n, k\right) \bar{f}\left(n_{1}, n_{2}-1, n, k\right)=-1 .
\end{aligned}
$$

Из (42), (56), (60) и (61) следует, что функция $f\left(n_{1}, n_{2}, n, k\right)$ с необходимостью представима в виде

$$
f\left(n_{1}, n_{2}, n, k\right)=(-1)^{n_{1}+n_{2}} f(n, k)
$$

где функция $f(n, k)$ должна удовлетворять условию

$$
f(n, k) \bar{f}(n, k)=1 \text {. }
$$

Теперь надо исследовать, имеют ли оставшиеся операторы $\left(b^{\prime}, b^{\prime \prime}, L_{+}, L_{-}\right)$одинаковый вид в терминах $\left(a, a^{*}\right)$ и $\left(b, b^{*}\right)$. Мы рассмотрим только оператор $b^{\prime}$, поскольку вычисления с другими операторами аналогичны (и проще).

Из (4) и (20) следует, что

$$
\begin{aligned}
b^{\prime} e\left(n_{1}, n_{2}, n, k\right)= & b^{\prime}\left(a_{1}^{\prime \prime}\right)^{n_{1}}\left(a_{2}^{\prime \prime}\right)^{n_{2}} e_{n k}= \\
= & \left(a_{1}^{\prime \prime}\right)^{n_{1}} b^{\prime}\left(a_{2}^{\prime \prime}\right)^{n_{2}} e_{n k}+n_{1}\left(a_{1}^{\prime \prime}\right)^{n_{1}-1} L_{+}\left(a_{2}^{\prime \prime}\right)^{n_{2}} e_{n k}= \\
= & \left(a_{1}^{\prime \prime}\right)^{n_{1}}\left(a_{2}^{\prime \prime}\right)^{n_{2}} b^{\prime} e_{n k}+n_{2}\left(a_{1}^{\prime \prime}\right)^{n_{1}}\left(a_{2}^{\prime \prime}\right)^{n_{2}-1} L_{-} e_{n k}+ \\
& +n_{1}\left(a_{1}^{\prime \prime}\right)^{n_{1}-1}\left(a_{2}^{\prime \prime}\right)^{n_{2}} L_{+} e_{n k}+n_{1} n_{2}\left(a_{1}^{\prime \prime}\right)^{n_{1}-1}\left(a_{2}^{\prime \prime}\right)^{n_{2}-1} b^{\prime \prime} e_{n k} .
\end{aligned}
$$


С помощью формул (12) можно выразить действие операторов $b^{\prime}, b^{\prime \prime}, L_{+}, L_{-}$на минимальные векторы в терминах операторов $B$ :

$$
\begin{aligned}
b^{\prime \prime}= & B^{++}+a_{1}^{\prime \prime} B^{-+}\left(h_{1}-1\right)^{-1}+a_{2}^{\prime \prime} B^{+-}\left(h_{2}-1\right)^{-1}+ \\
& +a_{1}^{\prime \prime} a_{2}^{\prime \prime} B^{--}\left[\left(h_{1}-1\right)\left(h_{2}-1\right)\right]^{-1}, \\
L_{+}= & B^{+-}+a_{1}^{\prime \prime} B^{--}\left(h_{1}-1\right)^{-1}, \\
L_{-}= & B^{-+}+a_{2}^{\prime \prime} b^{\prime}\left(h_{2}-1\right)^{-1}, \quad b^{\prime}=B^{--},
\end{aligned}
$$

а затем воспользоваться (23).

Таким образом, можно явно вычислить $b^{\prime} e\left(n_{1}, n_{2}, n, k\right)$ в (64), найти с помошью (28) матричные элементы $b^{\prime}$ и, пользуясь $(32)$, записать оператор $b^{\prime}$ во вторично-квантованной форме. В результате получаем

$$
\begin{aligned}
b^{\prime} & =\sum_{n=0}^{n_{\max }-1} \sum_{k=0, s} \sum_{n_{1}=1}^{N_{1}} \sum_{n_{2}=1}^{N_{2}} \frac{n_{1} n_{2} a^{*}\left(n_{1}-1, n_{2}-1, n+1, k\right) a\left(n_{1}, n_{2}, n, k\right)}{\operatorname{Norm}\left(n_{1}, n_{2}, n, k\right)}+ \\
& +\sum_{n=1}^{n_{\max }} \sum_{k=0, s} \sum_{n_{1}=0}^{N_{1}} \sum_{n_{2}=0}^{N_{2}} \frac{\left(n+s-k+n_{1}\right)\left(n+k+n_{2}\right) a^{*}\left(n_{1}, n_{2}, n-1, k\right) a\left(n_{1}, n_{2}, n, k\right)}{\operatorname{Norm}\left(n_{1}, n_{2}, n, k\right)}+ \\
& +\sum_{k=0}^{s-1} \sum_{n_{1}=0}^{N_{1}} \sum_{n_{2}=1}^{N_{2}} \frac{\left[n_{2}\left(s-k+n_{1}\right) /(s-k)\right] a^{*}\left(n_{1}, n_{2}-1,0, k+1\right) a\left(n_{1}, n_{2}, 0, k\right)}{\operatorname{Norm}\left(n_{1}, n_{2}, 0, k\right)}+ \\
& +\sum_{k=1}^{s} \sum_{n_{1}=1}^{N_{1}} \sum_{n_{2}=0}^{N_{2}} \frac{n_{1}\left(n_{2}+k\right)(s+1-k) a^{*}\left(n_{1}-1, n_{2}, 0, k-1\right) a\left(n_{1}, n_{2}, 0, k\right)}{\operatorname{Norm}\left(n_{1}, n_{2}, 0, k\right)}
\end{aligned}
$$

где $n_{\max }=p-1-s, N_{1}=N_{1}(n, k)$ и $N_{2}=N_{2}(n, k)$ (см. раздел 3$)$.

Следуюший шаг состоит в том, чтобы с помощью (35) и (36) выразить операторы $\left(a, a^{*}\right)$ через операторы $\left(b, b^{*}\right)$ и воспользоваться $(34),(41),(43)$ и $(62)$. В результате устанавливаем, что формула (66) имеет одинаковьй вид в терминах $\left(a, a^{*}\right)$ и $\left(b, b^{*}\right)$, только если

$$
f(n, k)=c(-1)^{n},
$$

где $c$ - произвольная константа, удовлетворяющая условию $c \bar{c}=1$.

Аналогичные выгисления для операторов $b^{\prime \prime}, L_{+}, L_{-}$показывают, что если вьполнено условие (67), то эти операторы имеют одинаковый вид в терминах $\left(a, a^{*}\right)$ и $\left(b, b^{*}\right)$. Теперь из (41) и (62) следует, что окончательное решение для $\eta\left(n_{1}, n_{2}, n, k\right)$ имеет вид

$$
\eta\left(n_{1}, n_{2}, n, k\right)=\alpha f\left(n_{1}, n_{2}, n, k\right), \quad f\left(n_{1}, n_{2}, n, k\right)=(-1)^{n_{1}+n_{2}+n},
$$

где $\alpha$ удовлетворяет условию (43).

Мы доказали, что $A B$-симметрия, определенная посредством формулы (35), действительно является фундаментальной симметрией в КТПГ (по крайней мере, для безмассовых элементарных частиц). 


\section{7. ПРОБЛЕМА СУЩЕСТВОВАНИЯ НЕЙТРАЛЬНЫХ ЭЛЕМЕНТАРНЫХ ЧАСТИЦ}

Предположим теперь, что рассматриваемая частица нейтральна, т.е. совпадает со своей античастицей. На языке операторов $\left(a, a^{*}\right)$ и $\left(b, b^{*}\right)$ это означает, что данные наборы совпадают, т.е. $a\left(n_{1}, n_{2}, n, k\right)=b\left(n_{1}, n_{2}, n, k\right)$ и $a^{*}\left(n_{1}, n_{2}, n, k\right)=b^{*}\left(n_{1}, n_{2}, n, k\right)$. Поэтому формула (35) теперь имеет вид

$$
a^{*}\left(n_{1}, n_{2}, n, k\right)=\frac{\eta\left(n_{1}, n_{2}, n, k\right) a\left(\tilde{n}_{1}, \tilde{n}_{2}, n, k\right)}{F\left(\tilde{n}_{1}, \tilde{n}_{2}, n, k\right)},
$$

и, следовательно,

$$
a\left(n_{1}, n_{2}, n, k\right)=\frac{\bar{\eta}\left(n_{1}, n_{2}, n, k\right) a^{*}\left(\tilde{n}_{1}, \tilde{n}_{2}, n, k\right)}{F\left(\tilde{n}_{1}, \tilde{n}_{2}, n, k\right)} .
$$

Из (41) и (43) вытекает, что эти выражения согласуются друг с другом, только если

$$
f\left(n_{1}, n_{2}, n, k\right) \bar{f}\left(\tilde{n}_{1}, \tilde{n}_{2}, n, k\right)= \pm 1
$$

где знак плюс относится к антикоммутаторам, а знак минус - к коммутаторам. Возникает вопрос о том, совместимы ли друг с другом соотношения (68) и (71). Из (21), (62), (63) и определения преобразования $n_{j} \rightarrow \tilde{n}_{j}$ (см. раздел 4 ) следует, что

$$
f\left(n_{1}, n_{2}, n, k\right) \bar{f}\left(\tilde{n}_{1}, \tilde{n}_{2}, n, k\right)=(-1)^{s} .
$$

Сравнивая формулы (71) и (72), заключаем, что они несовместимы друг с другом, если справедлива теорема о связи спина со статистикой. Поэтому безмассовые нейтральные частицы в КТПГ не могут быть элементарными, а могут быть только составными.

\section{8. ОБСУЖДЕНИЕ}

В данной статье были рассмотрены безмассовые НП в КТПГ. Одно из ключевых отличий КТПГ от стандартной теории заключается в том, что в КТПГ частица и ее античастица представляют собой разные состояния одного и того же объекта. Как следствие операторы уничтожения и рождения частицы и ее античастицы могут быть выражены друг через друга. Это накладывает дополнительные ограничения на структуру теории. В частности, формула (35) определяет новую симметрию, не имеюшую аналога в стандартной теории. В разделе 6 было показано, что это действительно симметрия в безмассовом случае, так как операторы представления имеют одинаковый вид в терминах операторов уничтожения и рождения для частиц и античастиц. В разделе 5 было также установлено, что новая симметрия согласуется с условием существования вакуума только для частиц с полуцелым спином (в обычных единицах). Как показано в разделе 7 , отсюда следует, что существование безмассовых нейтральных элементарных частищ в 
КТПГ несовместимо с теоремой о связи спина со статистикой. В отдельной статье будет показано, что эти результаты можно распространить и на массивный случай.

Является ли естественным то, что требование нормальной связи спина со статистикой запрешает сушествование нейтральных элементарных частиц? Если ограничения, накладываемые теоремой о связи спина со статистикой, отсутствуют, то нельзя исключить существования нейтральных элементарных частиц в КТПГ. Однако сушествование таких частиц представляется достаточно неестественным. Действительно, поскольку одно модулярное НП одновременно описывает частицу и ее античастицу, оператор энергии AdS обязательно содержит вклады от обеих частей спектра, соответствующих частице и ее античастице (см. формулу (52)). Если бы частица была тождественна своей античастище, то формула (52) содержала бы два равных вклада и, следовательно, значение энергии AdS было бы в два раза больше, чем нужно.

Хотя результат о несушествовании нейтральных элементарных частиц был получен как для бозонов, так и для фермионов, очевидно, что случай бозонов более важен. Возможность того, что фотон является составной частицей, уже обсуждалась в литературе. Например, в работах [16] исследовалась модель, в которой фотон состоит из двух дираковских синглетонов [17]. Однако в рамках стандартной теории составной характер фотона является лишь возможным (и привлекательным) сценарием, в то время как в КТПГ он является неизбежным.

Известно, что стандартная локальная квантовая теория поля (ЛКТП) достигла весьма впечатляюших успехов в плане согласия теории с экспериментом. В частности, квантовая электродинамика и теория электрослабых взаимодействий основаны на предположении, что фотон является элементарной частицей. Поэтому можно усомниться в том, что наше заключение имеет отношение к физике. В то же время в ЛКТП имеется несколько хорошо известных недостатков и внутренних противоречий. Большинство физиков считает, что ЛКТП следует принимать "как она есть", но в то же время что ЛКТП является “низкоэнергетическим приближением более глубокой теории, являющейся, возможно, даже не теорией поля, а чем-то иным, наподобие теории струн” [13].

В работах [3], [4] мы привели доводы в пользу того, что будущая квантовая физика будет основана на полях Галуа. При этом теория не содержит актуальной бесконечности, все операторы корректно определены, расходимостей не может быть в принципе и т.д. В то же время мы считаем КТПГ крайне привлекательной не только из-за этого.

На протяжении столетий ученые и философы пытались понять, почему математика столь успешно объясняет физические явления (см., например, [18]). Вместе с тем такая область математики, как теория чисел и, в частности, полей Галуа, практически не оказывает влияния на физику. Исторически каждая новая физическая теория обычно привлекала более сложную математику. Стандартными математическими инструментами современной квантовой теории являются дифференциальные и интегральные уравнения, распределения, аналитические функции, представления алгебр Ли в гильбертовых пространствах и т.д. В то же время весьма впечатляюшие результаты теории чисел о 
свойствах натуральных чисел (например, теорема Вильсона) и даже понятие простого числа вообше не используются! Читатель легко заметит, что КТПГ использует только арифметику полей Галуа (которые даже проще, чем множество натуральных чисел). Сама возможность того, что будушая квантовая теория могла бы быть сформулирована таким образом, представляет несомненный интерес.

\section{Список литературы}

[1] E. P. Wigner. Ann. Math. 1939. V. 40. P. 149.

[2] P. A. M. Dirac. Theory of electrons and positrons. In: The World Treasury of Physics, Astronomy and Mathematics. Ed. T. Ferris. Boston-New York-London: Little Brown, 1991. P. 80.

[3] Ф. М. Лев. ЯФ. 1988. Т. 48. С. 903; F. M. Lev. J. Math. Phys. 1989. V. 30. P. 1985; 1993. V. 34. P. 490

[4] F. M. Lev. Problem of constructing discrete and finite quantum theory. hep-th $/ 0206078$.

[5] Б. Л. Ван дер Варден. Алгебра. М.: Наука, 1979; K. Ireland, M. Rosen. A Classical Introduction to Modern Number Theory. Graduate Texts in Mathematics-87. New York-Heidelberg-Berlin: Springer, 1987; H. Davenport. The Higher Arithmetic. Cambridge: Cambridge Univ. Press, 1999.

[6] E. M. Friedlander, B. J. Parshall. Bull. Am. Math. Soc. 1987. V. 17. P. 129.

[7] C. Fronsdal. Rev. Mod. Phys. 1965. V. 37. P. 221.

[8] N. T. Evans. J. Math. Phys. 1967. V. 8. P. 170.

[9] B. Braden. Bull. Am. Math. Soc. 1967. V. 73. P. 482.

[10] H. Zassenhaus. Proc. Glasgow Math. Assoc. 1954. V. 2. P. 1.

[11] E. Inonu, E. P. Wigner. Nuovo Cimento. 1952. V. 9. P. 705.

[12] Ю. В. Новожилов. Введение в теорию элементарных частиц. М.: Наука, 1972.

[13] S. Weinberg. Quantum Theory of Fields. Cambridge: Cambridge Univ. Press, 1995.

[14] W. Pauli. Phys. Rev. 1940. V. 58. P. 116.

[15] W. Pauli. Exclusion principle, Lorentz group and reflection of space-time and charge. In: Niels Bohr and the Development of Physics. Ed. W. Pauli. London: Pergamon, 1955. P. 30; G. Gravert, G. Luders, G. Rollnik. Fortschr. Phys. 1959. V. 7. P. 291.

[16] M. Flato, C. Fronsdal. Lett. Math. Phys. 1978. V. 2. P. 421; L. Castell, W. Heidenreich. Phys. Rev. D. 1981. V. 24. P. 371; C. Fronsdal. Phys. Rev. D. 1982. V. 26. P. 1988.

[17] P. A. M. Dirac. J. Math. Phys. 1963. V. 4. P. 901.

[18] E. P. Wigner. The unreasonable effectiveness of mathematics in natural sciences. In: The World Treasury of Physics, Astronomy and Mathematics. Ed. T. Ferris. Boston-New York-London: Little Brown, 1991. P. 526. 